\title{
Withdrawal of long-term epoprostenol therapy in pulmonary arterial hypertension (PAH)
}

\author{
George Calcaianu', Mihaela Calcaianu², Matthieu Canuet', Irina Enache' and Romain Kessler' \\ 'Service de Pneumologie, Fédération de médecine translationnelle de Strasbourg, Nouvel Hôpital Civil, Université de Strasbourg, Strasbourg, France; ${ }^{2}$ Service de \\ Cardiologie, Nouvel Hôpital Civil, Université de Strasbourg, France
}

\begin{abstract}
Once initiated for pulmonary arterial hypertension (PAH), epoprostenol treatment usually needs to be delivered for an indefinite duration. It is possible that some participants could be transitioned from epoprostenol to oral therapies. We retrospectively evaluated eight PAH participants transitioned from epoprostenol to PAH oral drugs. The criteria for epoprostenol withdrawal were: (I) persistent improvement of clinic and hemodynamic status; (2) stable dose of epoprostenol for the last three months; and (3) the participant's preference for oral therapy after evaluation of risk-benefit. We evaluated the clinical, functional, and hemodynamic status at baseline, at withdrawal, and after the transition to oral PAH therapy. The transition was completed in all eight participants. Four participants had a complete successful transition (CT) with a stable clinical and hemodynamic course and four participants had a partial successful transition (PT) remaining stable clinically, with a mild hemodynamic worsening, but without need to re-initiate epoprostenol therapy. The four CT participants were treated with epoprostenol for a shorter period of time (CT group: $35 \pm 30$ versus PT group: $79 \pm 49$ months, $P=0.08$ ). Mean epoprostenol dosage was lower in the CT group (CT group: $15 \pm 1.5 \mathrm{ng} / \mathrm{kg} / \mathrm{min}$ versus PT group: $24 \pm 1 \mathrm{I} \mathrm{ng} / \mathrm{kg} / \mathrm{min}, P=0.09)$. Safe withdrawal of epoprostenol treatment and transition to oral PAH therapy was possible in a small and highly selected group of participants. The majority of these participants had a portopulmonary PAH or PAH associated to HIV infection.
\end{abstract}

\section{Keywords}

epoprostenol, pulmonary arterial hypertension, PAH, withdrawal, carbon monoxide diffusing capacity (DLCO), right heart catheterization, treatment

Date received: 28 January 2017; accepted: 10 March 2017

Pulmonary Circulation 2017; 7(2) 439-447

DOI: $10.1177 / 204589321770240$ I

\section{Introduction}

Pulmonary arterial hypertension $(\mathrm{PAH})$ is a progressive and chronic disease that results in right heart failure and ultimately death if untreated. Participants with severe PAH (World Health Organization [WHO] functional class [FC] III and IV) are referred for treatment with parenteral prostanoid agents (PGI2). ${ }^{1}$ The continuous intravenous infusion of epoprostenol produces symptomatic and hemodynamic improvement, as well as improved survival in idiopathic PAH (IPAH). ${ }^{2-5}$ Despite the benefits, epoprostenol is an expensive and complex treatment with a short half-life and pharmacologic instability, requiring a permanent central venous access, exposing the participants to thrombosis, infections or delivery system malfunctions. It is associated with multiple side effects; the sudden withdrawal of the epoprostenol can result in severe clinical worsening and death. ${ }^{2,6-8}$

Nowadays the development of oral drugs like endothelin receptor antagonists (ERA), phosphodiesterase 5 inhibitors (PDE5I), guanylate cyclase stimulators and selective prostacyclin-receptor agonists, provides an alternative option to intravenous prostacyclin. Previous case reports have shown that epoprostenol can be transitioned to oral therapy in highly selected participants with a clinical and hemodynamic stability at follow-up, ${ }^{9-13}$ but there is a lack of understanding of the

\section{Corresponding author:}

George Calcaianu, Service de Pneumologie, Nouvel Hôpital Civil, I place de l'Hôpital, 67000 Strasbourg, France.

Email: calcaianugeorge@gmail.com (c) (1) (5) Creative Commons Non Commercial CC-BY-NC: This article is distributed under the terms of the Creative Commons Attribution-NonCommercial 3.0 License (http://www.creativecommons.org/licenses/by-nc/3.0/) which permits non-commercial use, reproduction and distribution of the work without further permission provided the original work is attributed as specified on the SAGE and Open Access pages (https://us.sagepub.com/en-us/nam/open-access-atsage).
(C) The Author(s) 2017. Reprints and permissions: sagepub.co.uk/journalsPermissions.nav journals.sagepub.com/home/pul @SAGE 
factors that predict a successful transition and there are no guidelines to manage this process. The transition to oral therapy remains guided by a limited literature, especially in consideration of long-term outcomes after transition. ${ }^{13,14}$ Moreover, there is no information about the risks of an unsuccessful transition and if this is related to worse outcomes. We report our single-center experience of weaning epoprostenol to oral drugs (ERA or PDE5 inhibitors).

\section{Material and methods}

\section{Study design}

Our single-center study was conducted based on a retrospective review of data in the PAH registry of University Hospital of Strasbourg, from May 2002 to January 2014, to identify the participants withdrawn from epoprostenol and switched to oral therapy. This study complied with the Declaration of Helsinki and was approved by the Institutional Review Board of the French learned society for respiratory medicine - "Société de Pneumologie de Langue Française" (CEPR no. 2016-006).

The participants selected as appropriate for the transition from epoprostenol demonstrated: persistent improvement of clinical and hemodynamic status (WHO FC I or II, cardiac index $[\mathrm{CI}] \geq 2.5 \mathrm{~L} / \mathrm{min} / \mathrm{m}^{2}$ and lower level of pulmonary vascular resistance [PVR] and mean pulmonary arterial pressure [mPAP] under treatment), stable dose of epoprostenol for the last three months and participant preference for oral therapy after verifying the complete understanding of the risks and benefits of transitioning.

We used an institutional two-stage protocol for epoprostenol weaning. At first, epoprostenol was tapered gradually at home (dose reduction of $2-3 \mathrm{ng} / \mathrm{kg} / \mathrm{min}$ per week) until participants were at a dose of $6-8 \mathrm{ng} / \mathrm{kg} / \mathrm{min}$ or $\leq 30 \%$ of baseline dose. The oral therapy was added at least two months prior to the initiation of epoprostenol weaning and right heart catheterization (RHC) was performed prior to withdrawal of epoprostenol.

For security measures, the epoprostenol discontinuation was completed in intensive care unit and epoprostenol was titrated down at a rate of $1 \mathrm{ng} / \mathrm{kg} / \mathrm{min}$ every hour with a strict monitoring of clinical and hemodynamic status. After complete withdrawal, the participants remained in contact with the staff of the PAH unit and they were reevaluated clinically and underwent different examinations: six-minute walking test (6MWT); trans-thoracic echocardiographic; and RHC testing every two to three months. The participants with a successful transition (defined by no need to re-instate the epoprostenol treatment) to oral therapy and stable improvement of hemodynamic and clinical status were included in the complete successful transition group (CT), whereas those with a successful transition and stable clinical status but with a mild hemodynamic worsening (higher mPAP and PVR with lower CI after the transition) were included in the partial successful transition group (PT).
The participants were followed and evaluated retrospectively at baseline, at epoprostenol withdrawal, and three to 12 months after, between May 2002 and January 2014. At that point, eight participants had been transitioned from epoprostenol to specific oral therapy.

\section{Investigations}

RHC was made in participants who were clinically stable for the last four weeks. Cardiac output (CO) was determined by the Fick method using measured oxygen consumption in room air. Pulmonary vascular resistance was calculated by dividing mPAP minus PAWP by CO. Pulmonary function tests, arterial blood gases, alveolar-arterial gradient (A$\mathrm{aO} 2$ ), 6MWT, and plasma B-type natriuretic peptide (BNP) levels were obtained the week before RHC.

\section{Statistical analysis}

SPSS Statistics version 20 was used for all statistical analysis. Continuous variables were described by mean \pm SD. Significant differences at baseline, between the two subgroups (complete versus partial successful transition), defined in the "Results" section, were determined using Mann-Whitney U-test. The hemodynamic data, 6MWT and arterial blood gases, from the time of diagnosis to the first evaluation at epoprostenol withdrawal and second evaluation at three or 12 months after, were compared between the groups by the Kruskal-Wallis test.

\section{Results}

\section{Participant features prior to epoprostenol withdrawal}

Between 2002 and 2014 only eight participants (Table 1) were transitioned from epoprostenol to oral therapy (two participants to monotherapy and six participants to dual therapy) in our center. The details of the PAH treatment for each participant are presented in Table 2. For two participants, diagnosed in 2002 and 2004, i.v. epoprostenol in monotherapy was the first line of treatment. In just one case, a dual therapy (epoprostenol and ambrisentan) was started after the diagnosis. At the time of prostanoid withdrawal, four participants had a triple therapy and four others had a dual-therapy.

Between 2002 and 2014, 209 participants were on i.v. epoprostenol therapy in our PAH center. The median duration of the follow-up, after epoprostenol transition, was 6.6 months (range $=3-24$ months). The total follow-up period was 63.6 months (range $=8.5-121$ months).

Of the eight participants, four were already treated by oral therapy at the time of epoprostenol initiation. Mean age was $54 \pm 10$ years with a female predominance (seven women, one man). In terms of respiratory function, we found a mild decrease in DLCO (mean value $=59 \pm 18 \%$ ), a decrease in $\mathrm{PaO} 2$ (mean value $=69 \pm 9 \mathrm{mmHg}$ ), and an 
Table I. Demographic and hemodynamic characteristics at baseline.

\begin{tabular}{|c|c|c|}
\hline Demographic variables & CT group & PT group \\
\hline Age (years) & $55.5 \pm 8$ & $52 \pm 13$ \\
\hline Female & $3 / 4$ & $4 / 4$ \\
\hline Time of EPO Tx (months) & $79 \pm 49$ & $49 \pm 30$ \\
\hline $\begin{array}{l}\text { Maximal dose of EPO } \\
\operatorname{Tx}_{x}(\mathrm{ng} / \mathrm{kg} / \mathrm{min})\end{array}$ & $15 \pm 1.5$ & $24 \pm I I$ \\
\hline \multirow[t]{3}{*}{$\mathrm{PAH}$ etiology } & 2 PoPAH & I IPAH \\
\hline & $\begin{array}{l}\text { I PAH-CTD } \\
\text { (Still syndrome) }\end{array}$ & I PoPAH \\
\hline & I IPAH & 2 PAH-HIV \\
\hline Duration of disease (months) & $133 \pm 10$ & $74 \pm 58$ \\
\hline 6MWD (m) & $357 \pm 128$ & $34 I \pm 169$ \\
\hline NYHA class III or IV & $4 / 4$ & $3 / 4$ \\
\hline DLCO (\%pred) & $74.6 \pm 11.8$ & $46.7 \pm 12^{*}$ \\
\hline $\mathrm{PaO} 2(\mathrm{mmHg})$ & $74 \pm 6$ & $65 \pm 11$ \\
\hline $\mathrm{A}-\mathrm{aO} 2(\mathrm{mmHg})$ & $36 \pm 16$ & $44 \pm 9$ \\
\hline mPAP (mmHg) & $46 \pm 5$ & $54 \pm 12$ \\
\hline $\mathrm{CO}(\mathrm{L} / \mathrm{min})$ & $5.22 \pm 1.8 \mathrm{I}$ & $3.32 \pm 1.41$ \\
\hline $\mathrm{Cl}\left(\mathrm{L} / \mathrm{min} / \mathrm{m}^{2}\right)$ & $2.75 \pm 0.95$ & $2.12 \pm 0.62$ \\
\hline RAP (mmHg) & $9 \pm 8$ & $12 \pm 2$ \\
\hline PVR (Wood Units) & $9 \pm 4$ & $17 \pm 10$ \\
\hline $\mathrm{SvO}_{2}(\%)$ & $63 \pm 8$ & $44 \pm 20$ \\
\hline
\end{tabular}

increase in alveolar-arterial oxygen gradient $(\mathrm{A}-\mathrm{aO} 2)$ (mean value $=40 \pm 13 \mathrm{mmHg}$ ). The hemodynamic features at baseline for the eight participants were: $\mathrm{mPAP}=50 \pm$ $9.5 \mathrm{mmHg}, \quad \mathrm{CO}=4.3 \pm 1.8 \mathrm{~L} / \mathrm{min}, \mathrm{CI}=2.4 \pm 0.8 \mathrm{~L} / \mathrm{min} /$ $\mathrm{m}^{2}, \mathrm{PVR}=13 \pm 8$ Wood Units (WO), $\mathrm{SvO} 2=55 \pm 15 \%$. The mean duration of epoprostenol treatment was $57 \pm 45$ months.

In terms of PAH etiology, two participants were diagnosed with IPAH, two with PAH associated with HIV, three with porto-pulmonary hypertension, and one with PAH associated to connective tissue disease (Still syndrome) (Table 1).

From the three participants with porto-pulmonary PAH (PoPAH), two of them received a liver transplantation: the first participant developed the PoPAH in the early stages of viral (hepatitis $\mathrm{C}$ ) cirrhosis, and therefore pulmonary vascular disease was a contraindication for liver transplantation. PAH treatment (epoprostenol and sildenafil) was initiated in 2010. Hemodynamic status improved and the participant could undergo the liver transplantation. Following liver transplantion, pulmonary vascular disease dramatically improved and therefore epoprostenol withdrawal was initiated, immediately after liver transplantation. Subsequently, the PDE5I (sildenafil $60 \mathrm{mg} /$ day) was continued. This participant was included in the CT group.

The second case of PoPAH appeared after liver transplantation, performed for a primary biliary cirrhosis. A dual therapy of epoprostenol and ERA (ambrisentan $5 \mathrm{mg} /$ day) was initiated with a good improvement of clinical and hemodynamic condition, which permitted to start epoprostenol withdrawal nine months after initiation. Two months before epoprostenol withdrawal, a PDE5I (sildenafil $60 \mathrm{mg}$ /day) treatment was added. The oral bi-therapy of ambrisentan and sildenafil was continued after epoprostenol discontinuation. This participant was included in the CT group.

The third case of PoPAH was diagnosed in 2002 and was associated with a viral (hepatitis B) cirrhosis. At that time, the only treatment for PAH was i.v. epoprostenol and therefore prostanoid perfusion was started in December 2002.

Because of surprising clinical and hemodynamic improvement, in January 2009, the medical staff decided to start epoprostenol withdrawal by adding bosentan and sildenafil treatment. In October 2009, the epoprostenol was completely transitioned to oral therapy (bosentan $250 \mathrm{mg}$ /day and sildenafil $60 \mathrm{mg} /$ day). This participant was included in the PT group.

Two participants developed PAH associated to HIV infection. The first case was diagnosed in August 2003 and was treated with epoprostenol and bosentan in dual therapy. At the six-month evaluation, we observed a dramatical hemodynamic and clinical improvement which permitted to initiate epoprostenol withdrawal. A second oral therapy (sildenafil $60 \mathrm{mg} /$ day) was started two months before epoprostenol discontinuation and therefore the participant continued a dual oral therapy (bosentan and sildenafil) after epoprostenol discontinuation.

The second case of PAH-HIV was diagnosed in 2002 and a dual therapy by epoprostenol and bosentan was initiated. Ten years later, we noticed an important amelioration of clinical and hemodynamical status which led to the start of epoptostenol discontinuation. A second oral treatment (tadalafil $40 \mathrm{mg} /$ day) was initiated two months before prostacyclin withdrawal. The dual oral therapy (bosentan and tadalafil) was maintained after complete epoprostenol withdrawal. These two cases of PAH-HIV were included in the PT group.

The first case of IPAH was diagnosed in 2004. An oral monotherapy (bosentan $250 \mathrm{mg}$ /day) was started immediately. Twelve months later, i.v. epoprostenol was added to better control the disease. In 2012, we noticed a dramatic improvement of the hemodynamic status and epoprostenol treatment was therefore discontinued, by adding bosentan $250 \mathrm{mg} /$ day, in monotherapy. This participant was included in the PT group.

The second case of IPAH was diagnosed in 2003. An oral monotherapy was rapidly instated by bosentan. One year later, because of induced hepatotoxicity, the treatment with bosentan was replaced by sildenafil $60 \mathrm{mg} /$ day. In 2008, the participant was hospitalized in the ICU for severe right heart failure and therefore i.v. epoprostenol was initiated. In the following years, the clinical and hemodynamical status were improved under i.v. epoprostenol and sildenafil treatment. This is why, in 2014, we proceeded to downgrade the epoprostenol dosage; the transition to a dual 


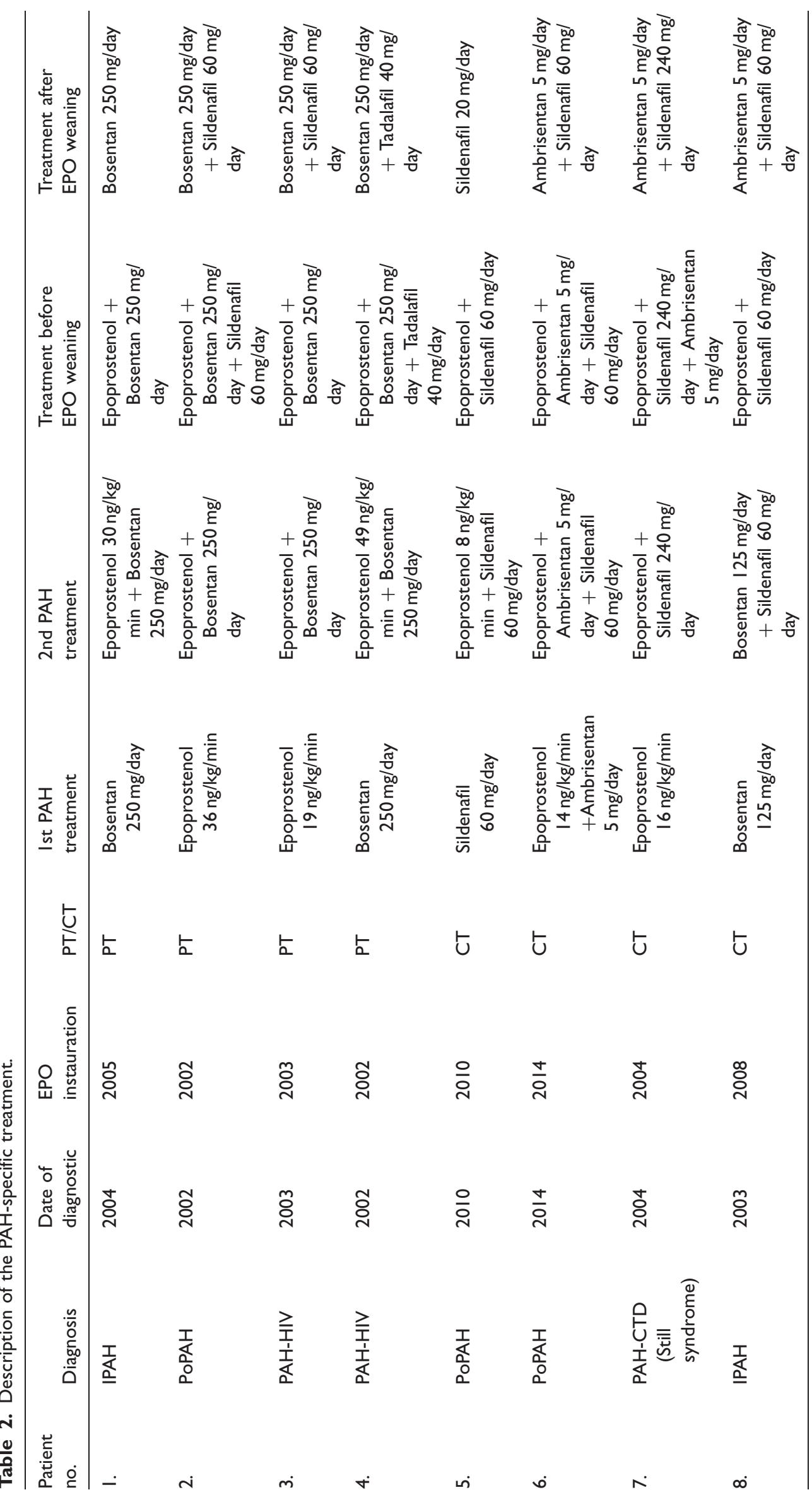


oral therapy (sildenafil $60 \mathrm{mg}$ /day and ambrisentan $5 \mathrm{mg} /$ day) was completed in July 2014. This participant was included in the CT group.

The eighth participant transitioned to oral therapy was diagnosed in 2004 by PAH associated with connective tissue disease (Still syndrome). The first line treatment was i.v. epoprostenol at a maximum dosage of $16 \mathrm{ng} / \mathrm{kg} / \mathrm{min}$; in the same participant, a corticoid therapy was initiated $(0.5 \mathrm{mg} / \mathrm{kg} / \mathrm{day})$. A few years later, the participant passed on dual therapy (epoprostenol and sildenafil) to better control the disease. In 2009, because of dramatic improvement in clinical and hemodynamic status, we proceeded to withdraw the prostanoid treatment by adding a second oral drug (ambrisentan $5 \mathrm{mg} /$ day) two months before. Finally, the epoprostenol was transitioned to a dual oral therapy with sildenafil (we used $240 \mathrm{mg}$ /day because the participant was included in the SUPER trial) and ambrisentan ( $5 \mathrm{mg} /$ day). This participant was included in the CT group.

\section{Transition characteristics}

The main reason to proceed for epoprostenol withdrawal was at the participant's request. No withdrawal was conducted because of intolerable epoprostenol side effects. In the PT group, we found one case of IPAH, one case of PoPAH, and two cases of PAH-HIV. The CT group was formed by one case of IPAH, one case of PAH associated to connective tissue disease (Still syndrome), and two cases of PoPAH. Five participants started the epoprostenol treatment before 2005, previously the era of oral specific PAH treatment.
The participants in the CT group tended to have a shorter duration of epoprostenol treatment prior to withdrawal, but the difference was not statistically significant (CT group: $35 \pm 30$ versus PT group: $79 \pm 49$ months, $P=0.083$ ). The maximal dose of epoprostenol tended to be lower in the CT group $(15 \pm 1.5$ versus $24 \pm 11 \mathrm{ng} / \mathrm{kg} / \mathrm{min}, \quad P=0.092)$ (Fig. 1). The duration of oral specific therapy before the transition was 33.5 months (range $=2-130$ months).

We also observed a normal level of DLCO for participants in the CT group $(75 \pm 12$ versus $47 \pm 12 \%, P=0.034)$. Table 3 shows the clinical and hemodynamic characteristics at the time of diagnosis, at epoprostenol withdrawal, and after complete discontinuation of prostanoid treatment. All eight participants were in WHO FC I or II when the decision of transition to oral therapy was taken. No significant differences were noted between the CT and PT groups for 6MWT, $\mathrm{PaO} 2, \mathrm{~A}-\mathrm{aO} 2, \mathrm{mPAP}, \mathrm{PVR}$, or $\mathrm{CI}$ at baseline (Table 1). At the time of epoprostenol weaning, we observed a trend of a longer six-minute walking distance (6MWD) $(581 \pm 47 \mathrm{~m}$ versus $316 \pm 175 \mathrm{~m}, P=0.083)$ and a lower mPAP ( $23 \pm 5$ versus $51 \pm 23, P=0.08)$ for participants in the CT group.

\section{Follow-up data after epoprostenol withdrawal}

All eight participants had a successful transition to oral agents. Four participants presented a stable clinical and hemodynamic status after transition (CT group). Four other participants were stable clinically but they experienced a mild hemodynamic worsening after epoprostenol withdrawal (PT group): mPAP and PVR increased slightly

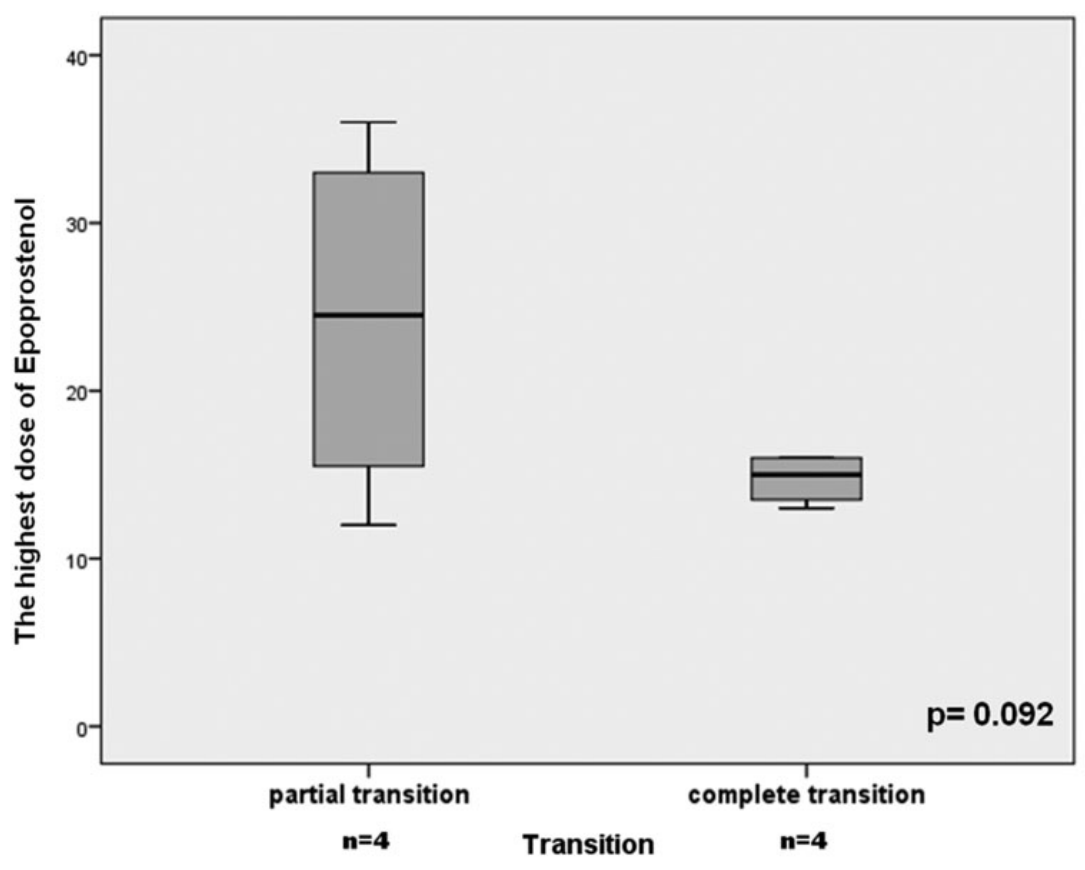

Fig. I. Comparison between the CT and PT group in terms of maximal dose of epoprostenol. 
from $45 \pm 15$ to $48 \pm 1 \mathrm{mmHg}(P=0.38)$ and $5 \pm 2$ versus $7.7 \pm 4 \mathrm{WU}$, respectively $(P=0.39)$. There were no significant differences (Table 3) in terms of clinical or hemodynamic status between the CT and PT groups throughout the follow-up $(P>0.05)$.

All eight participants had a clinical improvement at the time of treatment transition (WHO FC I or II) and they maintained this good clinical status for at least 19 months after epoprostenol withdrawal. To date, no deaths occurred and no participants were restarted with prostanoid treatment.

\section{Discussion}

Our study presents the clinical experience of our specialized PAH center in epoprostenol transition to oral therapy, based on a predetermined weaning protocol for a highly selected group of participants with the following features: persistent improvement of clinical and hemodynamic status (WHO FC I or II, CI $\geq 2.5 \mathrm{~L} / \mathrm{min} / \mathrm{m}^{2}$, lower $\mathrm{mPAP}$ and PVR); stable dose of epoprostenol for the last three months; and participant preference for oral therapy after evaluation of risk-benefit. There is currently no consensus about which participants should be candidates for transitioning to oral agents and such attempts remain guided by case reports or small case series. ${ }^{10,12,13,15,16}$ Our findings indicate that selected $\mathrm{PAH}$ participants, with stable clinical status (WHO FC I or II), with low doses of epoprostenol and persisting hemodynamic improvement, may be safely transitioned to oral drugs with satisfactory long-term results.

Successful withdrawal of epoprostenol treatment was first reported by $\mathrm{Kim}$ et al. ${ }^{9}$ in four participants: three with IPAH and one with scleroderma PAH. These participants presented a normalization of PAP and improved their WHO functional status to class I or II before the weaning procedure started. One of the largest cohorts of PAH participants transitioned from parenteral prostacyclin (i.v. epoprostenol or subcutaneous treprostinil) was reported by Suleman and Frost, in which 23 participants were included; 15 participants were able to wean from prostaglandin therapy to bosentan, although four participants had to resume the prostacyclin treatment within one month after withdrawal. All participants in this cohort had persistently elevated PAP, assessed only by echocardiography and were in WHO FC II or III.

Two more recent studies reported long-term results after epoprostenol withdrawal and transition to oral therapy (bosentan and sildenafil in monotherapy or combination). ${ }^{12,13}$ The percentage of participants who failed the transition to oral therapy was almost the same (approximately $30 \%$ ). These participants were able to reach their baseline clinical status after resumption of epoprostenol treatment but with persistently abnormal hemodynamics. Johnson et al. also showed a persistent clinical improvement for the participants with a normalized pulmonary 
hemodynamics ( $\mathrm{mPAP}<30 \mathrm{mmHg}$ and $\mathrm{PVR}<4 \mathrm{WU}$ ). The hemodynamic and clinical benefit obtained under epoprostenol treatment has persisted for up to 46 months after withdrawal, suggesting that epoprostenol therapy may be associated with sustained remodeling of pulmonary vasculature. Although this positive hemodynamic result after epoprostenol transition appears to be the exception. ${ }^{13}$ All the participants recruited in our report had a complete transition to oral therapy (bosentan or sildenafil in monotherapy or combination). Four participants (PT group) presented a deterioration of hemodynamic parameters with higher mPAP, PVR, and lower CI after epoprostenol transition, but with a sustained good clinical status (WHO FC I or II) and no cases of mortality. We found a significant difference between the CT and PT groups in terms of DLCO $(75 \pm 12 \%$ pred in the CT group versus $47 \pm 12 \%$ pred in the PT group, $P=0.034$ ), which could suggest that a complete successful transition was possible for the participants with a less severe disease. At the time the decision of transition was made, we observed a trend of a higher walking distance at 6MWD $(581 \pm 47 \mathrm{~m}$ versus $316 \pm 175 \mathrm{~m}$, $P=0.083)$ (Fig. 2) and a lower mPAP (23 \pm 5 versus $51 \pm 23, P=0.08$ ) (Fig. 3) for participants in the CT group.

It is uncertain if underlying disease could have an impact on the success rate of transition process. Previous studies concerning the transition from parenteral prostanoids to oral agents included IPAH, connective tissue disease (CTD)-associated PAH or systemic lupus erythematosus
(SLE)-associated PAH, with contradictory results regarding the underlying pathology. We succeeded the transition of two IPAH participants, two HIV-PAH participants, one CTD-PAH (Still syndrome), and three PoPAH; one PoPAH participant was diagnosed before liver transplantation and needed epoprostenol therapy to improve the pulmonary hemodynamic. After liver transplantation, the participant normalized the pulmonary vascular and right ventricular parameters with stable clinical status at WHO FC I. A second participant who needed a liver transplantation developed PoPAH after hepatic surgery, considered a rare complication of PAH de novo after organ transplantation. Although PAH was very severe at the time of diagnosis, we observed a drastic and rapid improvement of hemodynamic and clinical features which permitted the withdrawal of epoprostenol six months after initiation. Two of the PoPAH participants were transitioned to ERA in monotherapy or dual therapy, without any safety issues; there was no increase in hepatic enzyme levels nor other side effects.

Finally, just two cases from eight, were diagnosed with IPAH. We believe that the etiology of PAH can explain the successful of transition and therefore this series of cases is not representative for WHO Group $1 \mathrm{PAH}$.

The variables reported in the previous studies which could have a role in predicting the success or failure of prostanoids withdrawal were: maximum prostanoid dose; duration of prostanoid therapy; and duration of oral

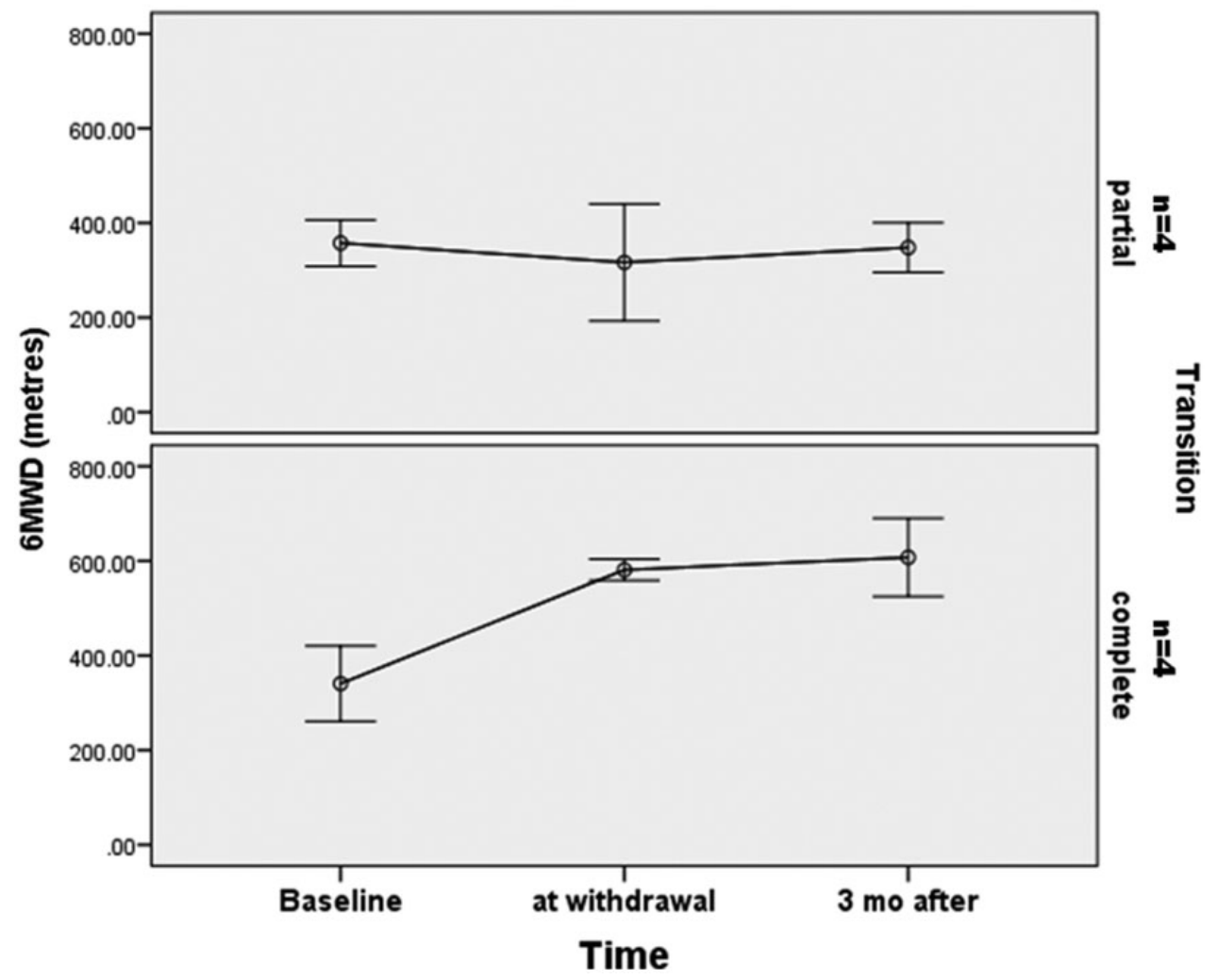

Fig. 2. Evolution of 6MWD from baseline to epoprostenol withdrawal and three months after. 


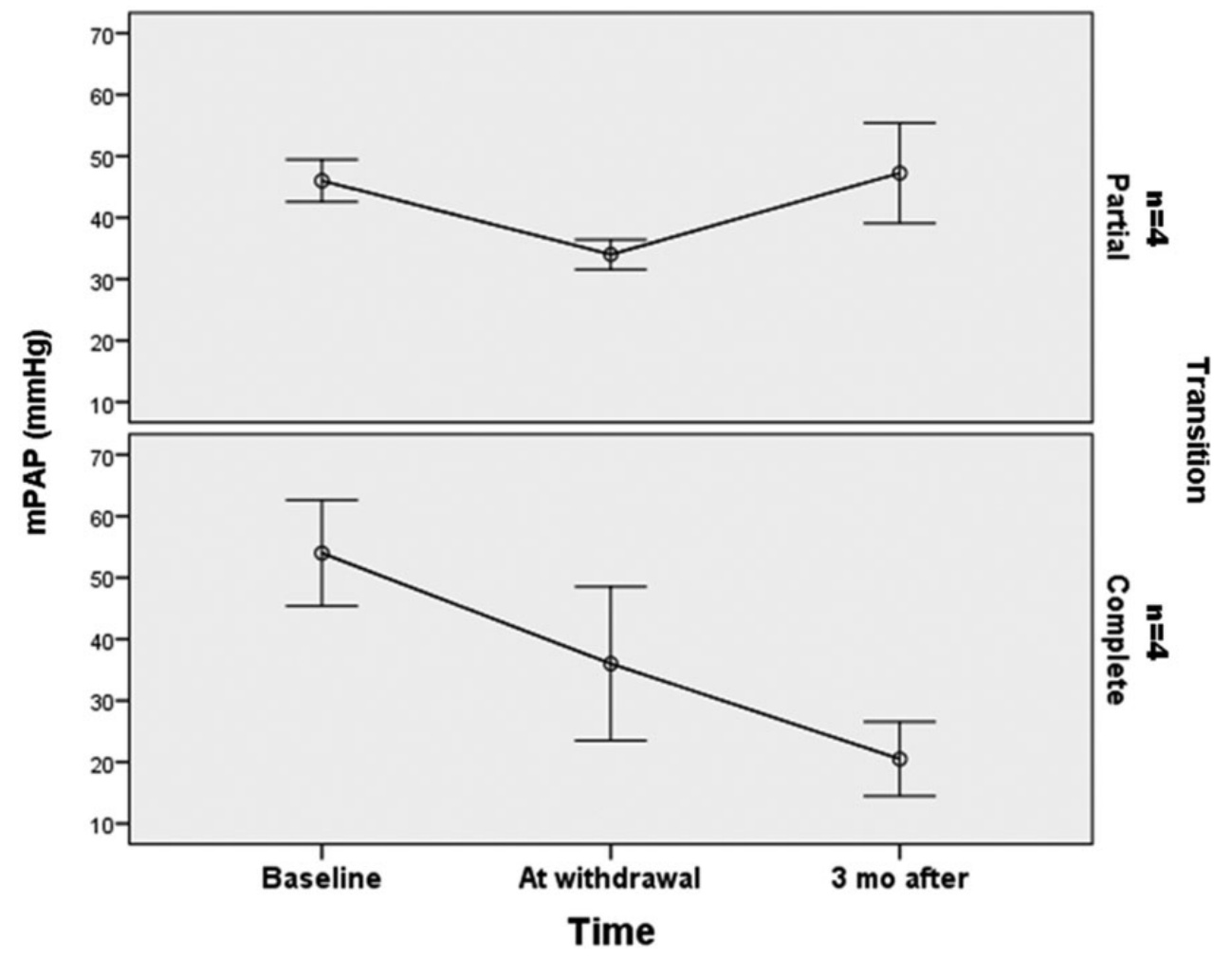

Fig. 3. Evolution of mPAP from baseline to epoprostenol withdrawal and three months after.

therapy. ${ }^{10,14}$ In our study, the mean duration of epoprostenol treatment before transition to oral drugs was shorter in the group with a complete successful transition (CT group $35 \pm 30$ versus PT group $79 \pm 49$ months, $P=0.083$ ). This could suggest that participants in CT group presented a less severe PAH. There are contradictory results in the literature of prostanoids withdrawal; if Steiner et al.${ }^{14}$ observed that longer duration of prostanoid treatment might be linked to a successful weaning, Suleman et al. ${ }^{10}$ found an increased trend for longer duration of prostanoid treatment for the participants who failed the transition to oral drugs.

Four participants were on triple therapy at the time of prostanoid discontinuation and they were distributed in the same proportion to the CT or PT groups. The other four participants had a dual therapy at the time of epoprostenol weaning. The therapeutic strategy (triple versus dual therapy at the time of transition) did not show any difference at follow-up, in terms of hemodynamic or clinical status.

Few studies suggested that a lower maximal prostanoid dose may be associated with better outcomes and higher weaning success since this may reflect a less severe disease. ${ }^{10,14} \mathrm{We}$ found a tendency of lower maximal epoprostenol dose for the participants with a complete successful transition $(15 \pm 1.5$ versus $24 \pm 11 \mathrm{ng} / \mathrm{kg} / \mathrm{min}, P=0.092)$.

In our study, the participants with a partial successful transition did not experience a severe hemodynamic or clinical deterioration after epoprostenol withdrawal and there was no mortality due to this transition attempt.
This study has several limitations. This was an observational, retrospective study on a very small population. The small number of participants did not allow a statistical analysis with enough power. It is not clear if these participants will sustain the clinical and hemodynamic benefit on the long term, especially for the PT group of participants.

In conclusion, carefully selected PAH participants can be safely transitioned from epoprostenol to oral drugs treatment, with long-term clinical stability. This high-risk transition process must be performed in PAH expert centers. Higher DLCO, lower maximal dose, and shorter period of epoprostenol treatment could predict a higher likelihood of successful prostanoid withdrawal. These findings require further confirmation in prospective clinical trials.

Current knowledge. Epoprostenol is recommended for the treatment of severe PAH. Once initiated, intravenous epoprostenol treatment usually needs to be delivered for an indefinite duration.

What this paper contributes to our knowledge. It is possible that some participants with a dramatic improvement of PAH can be transitioned from epoprostenol to oral PAH therapies. Higher DLCO, lower maximal dose and shorter period of epoprostenol treatment could predict a higher likelihood of successful prostanoid withdrawal.

However, there is a small proportion of IPAH transitioned to oral therapy. Therefore, we believe that some 
subtypes of PAH, like PoPAH or PAH-HIV, are more likely to be transitioned from i.v. epoprostenol to oral PAH drugs.

\section{Conflict of interest}

The authors declare that there is no conflict of interest.

\section{Funding}

This research received no specific grant from any funding agency in the public, commercial, or not-for-profit sectors.

\section{References}

1. Galiè N, Humbert M, Vachiery J-L, et al. 2015 ESC/ERS Guidelines for the diagnosis and treatment of pulmonary hypertension: The Joint Task Force for the Diagnosis and Treatment of Pulmonary Hypertension of the European Society of Cardiology (ESC) and the European Respiratory Society (ERS)Endorsed by: Association for European Paediatric and Congenital Cardiology (AEPC), International Society for Heart and Lung Transplantation (ISHLT). Eur Heart $J$ 2016; 37(1): 67-119.

2. Barst RJ, Rubin LJ, McGoon MD, et al. Survival in primary pulmonary hypertension with long-term continuous intravenous prostacyclin. Ann Intern Med 1994; 121(6): 409-415.

3. McLaughlin VV, Genthner DE, Panella MM, et al. Reduction in pulmonary vascular resistance with long-term epoprostenol (prostacyclin) therapy in primary pulmonary hypertension. $N$ Engl J Med 1998; 338(5): 273-277.

4. Sitbon O, Humbert M, Nunes $\mathrm{H}$, et al. Long-term intravenous epoprostenol infusion in primary pulmonary hypertension: prognostic factors and survival. J Am Coll Cardiol 2002; 40(4): 780-788.

5. McLaughlin VV, Shillington A and Rich S. Survival in primary pulmonary hypertension: the impact of epoprostenol therapy. Circulation 2002; 106(12): 1477-1482.

6. Kuhn KP, Byrne DW, Arbogast PG, et al. Outcome in 91 consecutive subjects with pulmonary arterial hypertension receiving epoprostenol. Am J Respir Crit Care Med 2003; 167(4): $580-586$.
7. Rubin LJ. Primary pulmonary hypertension. $N$ Engl $J$ Med 1997; 336(2): 111-117.

8. Robbins IM, Cuiper LL, Stein CM, et al. Angiotensin II mediates systemic rebound hypertension after cessation of prostacyclin infusion in sheep. $J$ Appl Physiol (1985) 1998; 85(2): 731-737.

9. Kim NH, Channick RN and Rubin LJ. Successful withdrawal of long-term epoprostenol therapy for pulmonary arterial hypertension. Chest 2003; 124(4): 1612-1615.

10. Suleman N and Frost AE. Transition from epoprostenol and treprostinil to the oral endothelin receptor antagonist bosentan in patients with pulmonary hypertension. Chest 2004; 126(3): 808-815.

11. Ivy DD, Doran A, Claussen L, et al. Weaning and discontinuation of epoprostenol in children with idiopathic pulmonary arterial hypertension receiving concomitant bosentan. Am J Cardiol 2004; 93(7): 943-946.

12. Diaz-Guzman E, Heresi GA, Dweik RA, et al. Long-term experience after transition from parenteral prostanoids to oral agents in patients with pulmonary hypertension. Respir Med 2008; 102(5): 681-689.

13. Johnson RF, Loyd JE, Mullican AL, et al. Long-term followup after conversion from intravenous epoprostenol to oral therapy with bosentan or sildenafil in 13 patients with pulmonary arterial hypertension. J Heart Lung Transplant Off Publ Int Soc Heart Transplant 2007; 26(4): 363-369.

14. Steiner MK, Preston IR, Klinger JR, et al. Conversion to bosentan from prostacyclin infusion therapy in pulmonary arterial hypertension: a pilot study. Chest 2006; 130(5): $1471-1480$.

15. Vachiéry J-L, Hill N, Zwicke D, et al. Transitioning from i.v. epoprostenol to subcutaneous treprostinil in pulmonary arterial hypertension. Chest 2002; 121(5): 1561-1565.

16. Keogh AM, Jabbour A, Weintraub R, et al. Safety and efficacy of transition from subcutaneous treprostinil to oral sildenafil in patients with pulmonary arterial hypertension. $J$ Heart Lung Transplant Off Publ Int Soc Heart Transplant 2007; 26(11): 1079-1083. 